\title{
¡CON NOSOTRAS NO!: La organización autónoma de mujeres indígenas de Tierras Bajas un prisma para entender el presente en Bolivia
}

\author{
Claudia Cuellar \\ Universidad de Chile
}

\begin{abstract}
RESUMEN
Decir y sentir desde un nosotras hace visible la re-composición de la dominación en Bolivia y el lanzamiento de la acumulación por despojo en las tierras bajas. Además, implica hacer evidente el establecimiento de pacto(s) patriarcal(es) entre diversas formas y actores, para así reforzar las jerarquías funcionales a los grupos dominantes. El reforzamiento de dicha jerarquía ha generado la necesidad y el deseo de desplegar procesos organizativos autónomos, "entre mujeres", impulsados principalmente por mujeres indígenas de tierras bajas, en este sentido, cuando las mujeres deciden organizarse, se presenta un sin número de mediaciones funcionales a que la amalgama capitalista, colonial y patriarcal se refuerce en territorios que se resisten a vivir bajo las reglas del capital, así mismo, las organizaciones de mujeres que empiezan a desplegarse- nunca exenta de tensiones- es capaz de visibilizar otra política, una política viva, que se sostienen en tramas diversas, construye de manera múltiple, y hoy por hoy, es capaz de reconocer el acumulado histórico y reactivar la memoria de la experiencia de luchas en femenino. El presente artículo pretende analizar como la amalgama capitalista, colonial y patriarcal, se re-edifica a través de alianzas patriarcales en Bolivia y ante eso, como se genera una capacidad importante desde las mujeres para impugnar, y reconocer un acumulado de fuerzas que activa la decisión de luchar juntas.
\end{abstract}

Palabras claves: Mujer. Pacto Patriarcal. Autonomía. Bolivia.

\section{¡CON NOSOTRAS NO! The autonomous organization of indigenous women of Tierras Bajas a prism to understand the present in Bolivia}

\begin{abstract}
Talking and feeling from us - as in "us women" - visibilize the re-composition of domination in Bolivia and the launching of accumulation by dispossession in the lowlands. Furthermore it implies making evident the establishment of patriarchal pact (s) among diverse forms and actors, in order to reinforce the functional hierarchies of the dominant groups. The reinforcement of this hierarchy has generated the need and desire to set up autonomous organizational processes among women, driven mainly by indigenous women from the lowlands, in this sense, when women decide to organize, there is a number of functional mediations to which the capitalist, colonial and patriarchal amalgam is reinforced in territories that resist living under the rules of capital, likewise, the women's organizations that are beginning to unfold - never free of tensions - are capable of making visible an other politics, an alive politics, that is sustained in different plots, it's build in multiple ways, and today, is able to recognize the historical accumulated and reactivate the memory of the experience of feminine struggles. The present article intends to analyze how the capitalist, colonial and patriarchal amalgam is re-edified
\end{abstract}

\footnotetext{
* Concepto acuñado por Mariana Menendez en: Menendez, M. 2017. Entre mujeres: nuestro deseo de cambiarlo todo. En Apantle (págs. 53-68). Puebla: SOCCE.
} 
through patriarchal alliances in Bolivia and before that, how an important capacity is generated from women to challenge, and recognize an accumulated force that activates the decision to fight together. Keywords: Women. Patriarchal Pact. Autonomy. Bolivia. 


\section{LA QUE SE HACE VISIBLE CUANDO LAS MUJERES SE ORGANIZAN ENTRE SÍ}

"Yo le recordé que somos autónomas, es decir que nadie nos puede obligar si no queremos ser parte. Como mujeres indígenas y lideresas defendemos derechos de los pueblos de las Tierras Bajas, de las Mujeres Indígenas. Ahora nos quieren obligar a ser parte del MAS ${ }^{1}$. Nosotras no vamos a vender nuestra organización, no nos vamos a prestar a este juego sucio que ellos hacen como varones" CNAMIB $^{2}$

El reconocimiento de violencias múltiples emerge cuando nos juntamos a conversar mujeres que gestionamos la vida en la ciudad, las que gestionan la vida en los territorios indígenas y campesinos y las que transitan por ambos, sin embargo, la necesidad y urgencia - por cómo se estructura la dominación colonial y patriarcal - de la defensa de la vida en común ha generado que sean las mujeres indígenas las que inicien la tarea de romper con sus espacios mixtos y darse fuerza. Tomamos esa fuerza que narra una de las integrantes de la Confederación Nacional de Mujeres Indígenas de Bolivia- CNAMIB, para entender que acontece hoy en Bolivia.

En este texto lo que voy a sostener es que cuando las mujeres deciden romper con sus espacios orgánicos, reconocer su fuerza y abrir otro campo para impulsar esas decisiones, van también en ese movimiento habilitando un canal interpretativo de la realidad que estamos viviendo, es así que se genera un proceso de conciencia importante sobre la estructura que se va edificando a partir del despojo que vía disciplina miento patriarcal reactualiza la amalgama de dominación capitalista, colonial y patriarcal (Gutiérrez; Sosa; Reyes 2018: 2).

Pensar desde la reproducción de la vida, como aquel substrato de la vida social que el trabajo y esfuerzo de Silvia Federici alumbra con su desplazamiento,

desde el canónico sitio de la producción y reproducción del capital hacia el subversivo ámbito de la producción y reproducción de su "mercancía" más preciosa y necesaria: la fuerza de trabajo. Y, en dicho tránsito, alumbrar otras posibilidades para pensar la transformación social, la revolucionarización de lo existente, las luchas en defensa de la vida, de los medios de existencias y de las riquezas sociales todas, humanas y no humanas (Gutierrez 2015: 170).

Este proceso es clave, ya que los proyectos extractivistas y la acumulación por despojo que incrementan al capital, pone en crisis la forma en la que se produce y reproduce la vida en los territorios. Proceso que también responde a la crisis mundial de la reproducción social (Dinerstein

\footnotetext{
${ }^{1}$ Movimiento al Socialismo.

2 Extracto de noticia en el medio virtual: https://cambioclimatico.org.bo/contenido/nacio-la-organizacion-de-mujeresindigenas-guarani-de-bolivia-y-ya-enfrenta-varios-retos/
} 
2017) amplificando así, en primer término, una dominación patriarcal para el cimiento de dicha amalgama.

En este contexto, por ejemplo, lo que acontece en las tierras bajas de Bolivia, es un impulso de múltiples proyectos capitalistas; Entre estos, se presentan lógicas de acumulación y despojo por el fortalecimiento agroindustrial, las hidroeléctricas y gestión de riego e infraestructura. Para ello, opera también una relación con el latifundio - que mantiene sus privilegios - en un contexto donde la capacidad de redituar del Estado se sostiene y profundiza en las extracciones de minerales, petróleo y gas. Lo que significa entonces, fracturas en los territorios donde habitan mujeres y hombres que han sabido sostener una relación autónoma - respecto al capital - en sus territorios.

Una práctica política que ha sostenido el progresismo boliviano es aquella que el Estado instaura a través de una relación especifica con las organizaciones matrices indígenas - constituidas en la lucha por la tierra que se inicia en los años ochenta ${ }^{3}$ - como una política de pactos, alianza, que se gestiona en dos vías, por un lado con las organizaciones que en el periodo previo a la Asamblea Constituyente le otorgaron capacidad política al gobierno del MAS, pero que las prácticas y formación de Estado ha devenido en clientelización de las mismas, y por otro lado, aquellas que mantiene para sostener su capacidad de capturar excedente, y es con el poder terrateniente agroindustrial actual. En este sentido, volvemos a aquello que nos presenta Silvia Federici (Federici 2004) cuando analiza el orden político necesario para la instauración del capital, es decir, al no existir una clase burguesa hegemónica se establecen aquellos acuerdos entre el Estado, el clero y la burguesía emergente - como contra revolución - ideas que se sostendrán más adelante cuando desglosemos las luchas de mujeres.

En Bolivia, lo que se presenta hoy en día es el Estado y su partido de gobierno estableciendo alianzas, muy parecido al trabajo que realizó el MNR desde el año 1952, pero el proyecto del MAS si ha tenido la capacidad, sobre todo, en tierras bajas de ingresar con esta política de alianzas a las organizaciones indígenas. Entonces se hace visible la dominación que Tapia (2018) denomina como un acumulado histórico de la política extractivista del país, muy eficaz, porque sostiene y se sostiene a través del partido del MAS para lograr su consolidación.

Es así, aunque los territorios indígenas de las tierras bajas conviven desde hace mucho tiempo con lógicas de despojo, la fuerza de sus espacios organizativos había logrado gestionar relaciones estratégicas con el Estado y el capital, que eran para el sostenimiento de la vida común. Sin embargo,

\footnotetext{
${ }^{3}$ Para entender mejor sobre las luchas indígenas de las tierras bajas de Bolivia, se puede revisar Balza. 2001. Tierra, Territorio Territorialidad. Indigena. Un estudio antropologico sobre la evolución en las formas de ocupación del espacio del pueblo indigena chiquitano de ex reduccion jesuita de San José de Chiquitos. Santa Cruz: APCOB/SNV/IWGIA.
} 
hoy la intensificación del extractivismo y el régimen que impone, instala la dominación de forma más profunda.

En los últimos años se puede observar, por ejemplo, que en el 2014 y con los últimos acuerdos realizados entre el gobierno y la élite agro industrial, se amplía la frontera agrícola de 250.000 hectáreas, al millón 300 mil hectáreas para el cultivo de soya transgénica, y así el uso de aproximadamente once millones de agroquímicos y 20 millones de litros de diésel, con el fin de generar la biotecnología paradójicamente para la elaboración de biodiesel ${ }^{4}$.

El fortalecimiento agro industrial también viene acompañado de una avanzada de proyectos carreteros (el caso más importante tiene que ver con la resistencia levantada por el TIPNIS en el año 2011) e hidroeléctricas en el país. En la Amazonía boliviana, son tres mega represas las que están por construirse y por las cuales se han reorganizado resistencias, como ser las Hidroeléctricas Chepete, El Bala, Cachuela Esperanza y la construcción de la Hidroeléctrica Rositas, esta última en la cuenca del río Grande, que según el discurso aportará por lo menos 400 megavatios de energía para la exportación, asegura el gobierno de Bolivia que invertirá mil millones de dólares en la mega-obra. ${ }^{5}$

Rositas forma parte de un complejo de siete hidroeléctricas que se instalarán en la cuenca del río Grande y que abarcarán los departamentos de Santa Cruz, Cochabamba y Chuquisaca. Estos complejos hidroeléctricos, que ya están en proceso de licitación y construcción, son parte de un mega proyecto de desarrollo denominado "corazón energético" el cual pretende integrar al país a Sudamérica a través de la exportación de energías.

A este acumulado de proyectos se le suma el Decreto Supremo 2366 por el cual se aprobó la exploración de hidrocarburos dentro de las áreas protegidas de Bolivia, así se permite el ingreso a las reservas naturales para la extracción de petróleo y el proceso de consulta previa se convierte en un mero procedimiento administrativo.

Entonces, la amalgama patriarcal, capitalista y colonial que se reconstituye en Bolivia, a través de alianzas entre agroindustria, rentismo de Estado, partido de gobierno, intereses transnacionales, hoy imponen una jerarquización sostenida por este pacto - que es también - patriarcal pues instala un orden a través de mediaciones, que se ensaña en destruir las lógicas y flujos comunitarios y populares que garantizan la reproducción de la vida en los territorios, que se refuerza con violencia hacia los cuerpos de las mujeres para su fijación y clausura.

Una condición esencial y fundante del pacto, es entender el proceso desorganizador de

\footnotetext{
${ }^{4}$ Mayor información en: https://es.mongabay.com/2019/04/bolivia-gobierno-soya-transgenica/

${ }^{5}$ Referencia de las ultimas noticias al respecto en: https://es.mongabay.com/2018/11/conflictos-en-la-amazonia-de-bolivia/ 
las fuerzas comunes que sostienen la vida en los territorios en un contexto extractivista, ¿Cómo? Cuando las mujeres se reúnen y dan fuerza a su voz, lo primero que ponen como cuestión e impugnación es la violencia a la que están siendo expuestas en sus espacios mixtos, se busca el cercamiento, a través de la clausura en la participación de las decisiones, que ya no son comunes (asambleas), y también se las desplaza de la capacidad de mantener el trabajo colectivo para la reproducción autónoma de sus medios de vida, por la reorganización de la división sexual del trabajo que el capital (en este caso extractivista) instaura en los territorios.

Cuando Rita Segato (Segato 2016) plantea la idea de pirámide invertida como el cimiento patriarcal y como este se sostiene sobre el cuerpo femenino, su fijación y su capacidad que contiene la amalgama para estructurarse sobre él, y de ahí emanar poder, ayuda a nombrar como el patriarcado entonces reorganiza la dominación. Por tanto, según la experiencia viva de las mujeres amenazadas por el despojo en sus territorios identifican múltiples complicidades masculinas que se reinstalan para que ellas no sean capaces de disputar la gestión de la vida común.

Con las ideas que vamos planteando queremos identificar como la reorganización del poder "desde arriba" afecta la gestión de la vida autónoma en los territorios, partimos entonces de una serie de impugnaciones y deseos que las mujeres en sus diversas estrategias y escalas están realizando. Para ello reconocemos tres casos, uno que opera a nivel nacional desde la Confederación Nacional de Mujeres Indígenas de Bolivia (CNAMIB) que tiene mayor fuerza en las tierras bajas, para luego también dar cuenta de procesos de determinación de deseos que vienen gestionando las mujeres en dos territorios amenazados por el extractivismo. Territorio Guarayo y Territorio Guaraní, en la Asamblea de Mujeres del Pueblo Guaraní y la Central de Mujeres Indígenas de Guarayos, ambos en el departamento de Santa Cruz, Bolivia.

\section{2. "CON NOSOTRAS NO"}

Fue el conflicto del TIPNIS, que detona en el año 2011 y que hace evidente la avanzada geopolítica que impulsa el extractivismo sobre las tierras bajas de Bolivia, lo que pone en mayor crisis las organizaciones políticas que emergen desde 1980 en los Pueblos Indígenas en la lucha por el territorio. Si bien la Confederación de Pueblos Indígenas de Bolivia - CIDOB es una de las organizaciones que va impulsar la Asamblea Constituyente en el año 2005, y muestra un diálogo con la vertiente institucional 
de las luchas sociales, es el momento en que se rompe el Pacto de Unidad $^{6}$ por la defensa de los territorios amenazados por el extractivismo, y cuando formalmente la CIDOB y la CONAMAQ ${ }^{7}$ hacen un quiebre importante con la CONALCAN - que se presenta de manera intensiva el proceso de desorganización vía persecución y violencia, como también, cooptación principalmente a través de alianzas masculinas dominantes en las organizaciones sociales en las tierras bajas de Bolivia.

Si bien no nos interesa en este artículo especificar la relación Estado y organizaciones sociales que se inaugura en ese tiempo, es importante explicitar que es en ese momento que la crisis de la reproducción de la vida se impondrá con una forma específica - la patriarcal - sobre los territorios de las tierras bajas y es a partir de allí que las mujeres deciden organizarse e impugnar desde diversos espacios.

La Confederación Nacional de Mujeres Indígenas de Bolivia - CNAMIB, que ya se funda en el año 2007, empieza a desplegar con mayor fuerza la separación de aquellas organizaciones que responden al partido de gobierno desde el 2011, y son ellas quienes tejen y retejen los lazos con las luchas de las mujeres contra el despojo, por ello son las primeras en impugnar la política de cooptación y persecución que se empieza a desplegar contra ellas y sus territorios.

"Denunciamos al mundo que el gobierno boliviano busca apresar a los dirigentes indígenas para entregarles el TIPNIS a la Explotación de Transnacionales, pisoteando los derechos de los Pueblos Indígenas" pronunciamiento del año 2013 como denuncia importante que ellas estaban haciendo visible.

Esta organización macro regional, ha servido para que las mujeres que se encuentran en los territorios y viven en diversas escalas las transformaciones realizadas cuando el extractivismo activa la amalgama capitalista, colonial y patriarcal, pueden aliarse y reconstituir un espacio que las habilite a hablar. Se reorganizan a través de la impugnación que ha servido para poner los límites claros sobre como los varones están estableciendo sus alianzas e impulsan una forma política que no contiene sus deseos por garantizar la autonomía para reproducir su vida en los territorios.

En los territorios, con nuestras normas propias estamos trabajando con nuestros compañeros, hay una diferencia entre las orgánicas y nuestros espacios comunitarios, si hablamos de la gestión del territorio son las mujeres las que asistimos a las reuniones, las mujeres también

\footnotetext{
${ }^{6}$ Pacto de Unidad es una alianza nacional en evolución de las organizaciones de base de Bolivia que apoyan los derechos indígenas y agrarios, la reforma agraria, la reescritura de la Constitución de Bolivia 1967 a través de una Asamblea Constituyente y una transformación izquierda-indígena del Estado boliviano. Desde 2005, el Pacto ha sido un aliado cercano del presidente boliviano Evo Morales, y forma el núcleo de la Coordinación Nacional para el Cambio, una alianza pro-gobierno. En 2011, CONAMAQ y CIDOB se retiraron del Pacto. Ambas organizaciones sufrieron disputas internas en los años que siguieron. Una facción de cada división, alineada con el gobierno de Morales, volvió a unirse al Pacto en 2013.

${ }^{7}$ Pronunciamiento CNAMIB, en su página web: https:/www.facebook.com/Cnamib/
} 
están más en el ejercicio de la política, sobre todo por todo lo que ha pasado ya no hay mucha credibilidad en el varón sobre todo cuando a los hombres cuando se van a las organizaciones y los partidos políticos." (Entrevista Wilma Mendoza de la CNAMIB).

Otra impugnación y alianza importante entre mujeres emerge también cuando ya en el año 2016, vuelven a hacer visible la dominación que se reinstala contra ellas, ejemplo claro el pronunciamiento que sacan en contra de la CIDOB y su forma de gestionar las redes clientelares y patriarcales de poder al momento de instalar un proyecto hidroeléctrico contra seis comunidades, dos de ellas guaraní y que empiezan a activar la resistencia contra el despojo a través de diferentes estrategias:

CIDOB
CNAMIB $^{9}$ - ASAMBLEA DE MUJERES GUARANI (pronunciamiento junio de 2018)

Apoyo a la revolución Democrática y Cultural porque existe Rechazo total a las acciones del gobierno mediante el un proyecto nacional de Estado que incluye a los Pueblos Órgano Ejecutivo y Ministerio de Energías, la Empresa Indígenas de Tierras Bajas como parte del Estado.

de Energía-ENDE, que pretende desconocer las acciones legales realizadas por la resistencia al proyecto Apoyo a la revolución democrática porque, así como es hidroeléctrico Rositas. democrática liberal también es comunitaria.

Apoyo también en las elecciones del gobierno del MAS

Declaración como mujeres de vigilia permanente ante los atropellos del Estado Plurinacional y preocupación por los desastres que puede generar el proyecto hidroeléctrico.

Se suman a las acciones en defensa del territorio y la resistencia de las comunidades afectadas como Tatarenda y Yumao.

Junto con estos procesos por ejemplo, gracias a la fortaleza y la disputa por la autonomía que empezaron a impulsar las mujeres desde su organización autónoma, se registran también otros casos, por ejemplo la Central de Mujeres Indígenas Guarayas, que sufre una amenaza constante por el despojo agresivo por la minería ilegal, la des forestación y el narcotráfico denuncian como sus compañeros de las organizaciones cooptadas instauran procesos de negociación individual lo que se devuelve en violencia contra ellas e instala una política de acoso para que corporalmente ya no participen en el

\footnotetext{
8 Entrevista disponible en: https://www.servindi.org/actualidad-noticias-radioteca-audios/01/05/2016/bolivia-elmovimiento-indigena-desde-la-mirada-de

${ }^{9}$ La Confederación Nacional de Mujeres Indígenas de Bolivia CNAMIB, es una organización nacional, interlocutora valida de las Mujeres Indígenas de Tierras Bajas, en su relacionamiento con el entorno social, político y económico, a nivel local, regional, nacional e internacional que está orientada a la defensa de los derechos de las Mujeres Indígenas en el reconocimiento de su diversas identidades, de manera articulada a las políticas de la CIDOB, para promover el equilibrio en la relación entre hombres y mujeres y facilitar la participación activa y representativa en las decisiones políticas, económicas, sociales y culturales, a través del fortalecimiento de sus organizaciones de base, promoviendo la equidad y la vida plena de los Pueblos Indígenas.
} 
espacio de tomas colectivas de decisiones. ${ }^{10}$

En el mismo camino, los deseos de las mujeres guaraní se despliegan con la amenaza del proyecto hidroeléctrico Rositas, y es la agresión que reciben cuando se forma el Comité en Defensa de la Tierra y Territorio ${ }^{11}$ por la omisión y de hecho agresión que reciben por parte de la Asamblea del Pueblo Guaraní - APG, cuando la lucha empieza a impulsar mecanismos legales de defensas por lo que deciden fundar su propia Asamblea de Mujeres.

Es en estos procesos de organizar sus deseos y experiencias entre mujeres (Menendez, 2017: 129) donde las mujeres despliegan una fuerte impugnación anti patriarcal en primera instancia, pues deciden frenar la agresión que a sus cuerpos genera este proceso de cooptación que reestructura la amalgama.

\section{AGUJERAR LA REALIDAD PARA INTERPRETAR DESDE UN NOSOTRAS: EL PACTO PATRIARCAL}

Lo narrado anteriormente, como un momento de des-organización, cooptación e impugnación y el surgimiento de una política autónoma impulsada hoy desde las mujeres, despierta un momento interpretativo importante. Un ejercicio feminista sustancial es entender que cuando narramos los que nos pasa, no estamos nunca más desglosando una mirada sectorial de la vida - el rol que las políticas neoliberales nos han impuesto - pues el nombrar el mundo desde lo femenino es nombrar también desde esa parcialidad - la realidad toda.

María Milagros Rivera quien nos invita a pensar nuestro lugar en la historia y poder nombrarlo para visibilizar la autoría femenina y trenzar y dar cuenta de aquel conocimiento feminista, implica entonces reconocer todos los aportes "donde predomina un componente de lucha contra el orden simbólico patriarcal, y el femenino, en cambio, cuando se resalta la omisión del referente viril, el prescindir de ellos como medidas para conocer el mundo” (Rivera 2003: 27).

Partiendo de ese esfuerzo, en este apartado me remito a aquellas aportaciones feministas que buscan nombrar el orden patriarcal que sostiene la dominación, por tanto, propongo un breve diálogo entre algunas claves feministas y femeninas, que permiten alumbrar y reconocer las separaciones y fijaciones que la amalgama de dominación impone.

Construimos la idea de pacto patriarcal, con nociones que mujeres estudiosas nos han brindado

\footnotetext{
${ }^{10}$ Información obtenida a través de entrevistas realizadas por la investigadora.

11 Noticia en: https://www.noticiasfides.com/economia/rositas-tercer-juez-rechaza-accion-popular-de-guaranies-y-estosanuncian-ir-a-la-cidh--388103
} 
para entender el orden material y simbólico que se instaura contra nosotras, claro ejemplo Silvia Federici nos presenta la idea de "contra revolución" como una política de alianzas que instaura el capital para clausurar momentos importantes de luchas obreras y herejes donde las luchas de las mujeres toman una forma clara. Un ejemplo - que pervive - es a medida que las mujeres se organizan entorno a la defensa de los comunes, pareciese que los poderes tienen algo que los unifica, a medida que aparece las crisis de población - que podríamos llamarlas también como crisis reproductivas - se instaura un orden de dominio que se empeña en fijar a las mujeres. Este acuerdo que sostienen la acumulación histórica del capital, también se re actualiza en la manera que la que la acumulación también lo hace.

Pero ese pacto patriarcal que se instituye ya dentro del capital con el patriarcado del salario, tiene relación con como el Estado ha gestionado y tiene la intención, vía modernización, de seguir haciéndolo, pues tal como nos muestra la investigación de Gerda Lerner (Lerner 2017: 200), la idea de matrimonio, familia patriarcal como formas claves para la formación del Estado muestran como esta estructura para extender su dominio necesita, en primer lugar, imponer relaciones genéricas, donde la base de la propiedad en el núcleo familiar opera con el control de la sexualidad de las mujeres, la política de alianzas también se genera para la instalación del patriarcado cuando es el intercambio de mujeres lo que consolida - también - las relaciones de clases en la formación histórica del capital, que instala por tanto, un orden jerárquico "el colectivo masculino tiene un sin número de derechos sobre las mujeres que el colectivo femenino no tenía sobre los hombres" (Lerner 2017: 120), las mismas mujeres se convirtieron en un recurso que los hombres adquirían poder igual que se adueñasen de las tierras.

Esta búsqueda de apropiación del cuerpo femenino como máquina de reproducción necesaria para la formación de las clases, es lo que despliega y, en dialogo con Federici (2004) un sin número de resistencias desde las mujeres que se reactualizan - en la acumulación global del capital - bajo los despojos múltiples (Navarro 2017: 9) que ponen en crisis la reproducción de la vida. La acumulación del capital, instala entonces, un orden simbólico impuesto por el patriarcado colonial, a través de aquel pacto entre el "dios creador y el hombre en la tierra" como el único mediador, que desplaza y separa la creación de la procreación y a las mujeres de ser dueñas de sus propias creaciones (Gutiérrez; Sosa; Reyes 2018; Lerner 2017).

Otra idea importante para nombrar al patriarcado y su relación con el Estado moderno, viene desde las feministas, que parten de la crítica al contractualismo, para identificar el contrato sexual (Pateman 1995: 50), como pacto originario que constituye el inicio de una nueva sociedad civil que se “encuentra libre y sin las preocupaciones del Estado de naturaleza", argumenta que la parte 
fundamental del pacto social (y aquella que se oculta) es la igualdad de derecho de los varones de disfrutar del acceso sexual sobre las mujeres. Esto ocurriría a través de la lógica de inclusión-exclusión propia del pacto y de la posibilidad que tienen los hombres "libres" de replicar el pacto social con otras formas como el contrato de trabajo o el contrato matrimonial. Donde también se construye la idea de lo público, moderno, capitalista.

La crítica al contrato laboral y sexual, para la conformación del capital y del poder patriarcal, es porque muestra el sin número de alianzas que se reinstala ya en forma violenta y establecen los términos de las mediaciones en la amplitud de la dominación del Estado: "Según el andamiaje moderno, capitalista, patriarcal la historia de la esfera pública o esfera estatal no es otra cosa que la historia del género" (Segato 2016: 30).

Creemos que en la época actual en Bolivia, el Estado y su política clientelar, está relacionada con la idea desarrollista que el capitalismo necesita del Estado para su formación, por tanto, es condición necesaria la búsqueda de excedente y el establecimiento de mediaciones, imponiendo cada vez más la imposibilidad de las sociedades múltiples de darse forma (Gutierrez; Linsalata; Navarro 2016) y para nuestra hipótesis de trabajo esa capacidad de capturar excedente también vía acumulación opera sobre el control de la reproducción de la vida.

Entonces, lo que las mujeres están poniendo en evidencia es que ellas no son, ni estarán en ese pacto que se constituye. Este pacto patriarcal tiene una forma de operar tanto hacia los territorios para la acumulación, como también en la forma que se establece las relaciones dentro de él.

Sostenemos que el mismo instala una política de "fatria" (Pateman 2018: 24) que las feministas que analizan la formación de la burguesía ya también lo plantea, esta política de "fraters" de "hermanos" propio de los ideales liberales de igualdad, fraternidad y libertad, es el que se instala a la medida que el capitalismo reorganiza la vida social, "como un conjunto de relaciones sociales entre los hombres que tienen una base material, y aunque son jerárquicas, crean o establecen interdependencia y solidaridad entre ellos que los capacitan para dominar a las mujeres". Esta política de fatria es muy propia de la agro industria y oligarquía cruceña que gestiona todos los poderes para sí (Tomicha; Cuellar 2019) que instala esa forma de gestionar poder en sus acuerdos y pactos.

Las relaciones que se instalan dentro del pacto patriarcal genera una apariencia de individualización capitalista, pues opera en ese mecanismo de inclusión-exclusión, entonces el pacto siempre se sella por el intercambio de algo, en algunos casos el cuerpo femenino o en otros casos eso y los bienes naturales.

Almudena Hernando (2012) es capaz de identificar la "fantasía de la individualidad" por esa 
búsqueda incesante del capital de hacer invisible los vínculos, nos muestra también que la individualidad construida en torno a los hombres es la de la codependencia, es decir, gestionando relaciones de sumisión principalmente de las mujeres para el sostenimiento de la vida toda. Entonces aquello que se edifica desde arriba y que las mujeres indígenas están impugnando con mucha fuerza, es toda aquella forma de gestionar poder que impone el pacto patriarcal como centro de la acumulación que sostiene la dominación.

Pero, así como este pacto es cerrado y opera una serie de vínculos con aquellos varones - y algunas mujeres - que responden e ingresan a este pacto. "La política en femenino" (Gutierrez 2014) que están desplegando las mujeres muestra otra forma.

\section{POLÍTICA VIVA}

Tal como nos dice Ana Dinerstein (2017):

La construcción de la utopía concreta contiene una dimensión de creación y AFIRMACION inseparable de la de negación. La función utópica de la esperanza nos permite experimentar una vida mejor aun cuando no sepamos ni cómo ni cuándo. Nuestra conciencia anticipatoria nos permite soñar y crear nuevas realidades. En el momento de afirmación vemos la emergencia y consolidación de la política afectiva.

Es así que la interpretación antes realizada solo es posible desde el saber de la resistencia y la lucha, por tanto, existe una experiencia histórica sobre la autonomía que las mujeres hoy están poniendo en juego, esta experiencia de autonomía viene desde las luchas indígenas desplegadas en los lugares analizados, ellas están - desde un nosotras y un entre nosotras - ahora mostrando con claridad una diferencia cuando las luchas y las organizaciones que se institucionalizan se cooptan y subordinan al Estado.

La idea de autonomía de los Pueblos Indígenas como aquella capacidad de gestionar sus medios concretos de vida y su capacidad de decidir sobre los mismos, hoy se está empezando a reactivar desde estas luchas de las mujeres indígenas. Como mostramos anteriormente en los momentos en que ellas dicen con nosotras no, están habilitando su diferencia y reconociendo como construir desde allí.

Pero más allá de eso, la lucha por la reproducción de la vida con una fuerte impugnación desde la autonomía política, despliega una práctica política que por un lado se inscribe en los parámetros de la política viva, "como parte de la experiencia de lo cotidiano, que no responde a un programa ideológico, no representa a un sujeto único reconocible, y no busca una unidad, es una política de lo 
múltiple, autónoma, que no busca mediaciones, ni representaciones, que opera en el marco 'de lo que hay'”' (Gil 2011: 70). También se inscribe en el marco una política en femenino que remite a un modo de comprender lo político "como un lenguaje... un conjunto articulado de deseos y anhelos compartidos, de compromisos y prácticas colectivas que permiten a hombres y mujeres expresar lo que sentimos y pensamos, brindándonos la posibilidad de esperanzar, que es la condición necesaria de nuestro caminar..." (Gutierrez 2014: 30).

Por tanto, lo que van demostrando las mujeres indígenas en estos procesos, es que:

El sostén de la reproducción de las comunidades está en manos de la rebeldía que las mujeres están activando, es así que a mayor rebeldía mayor capacidad de sostener la vida en sus territorios. Esta política de la rebeldía, viene muy alineada con aquellos "deseos encarnados" deseos que se despliegan desde el cuerpo, los saberes y memorias que se activan desde allí (Briadotti 2018: 42).

Por otro lado, en estos procesos organizativos los vínculos que se empiezan a regenerar a través de la confianza buscan disolver el problema por el que se estructura la dominación colonial capitalista y es la búsqueda constante de la fijación de las diferencias para transformarlas en jerarquías. Pero las mujeres indígenas en esta organización están desplegando otra forma con sus propias tensiones, con vínculos de confianzas que se reconstituyen desde liderazgos locales y aquellos impulsados por resistencia anti-extractivistas con mujeres de distintas edades y trayectorias de vida diferenciadas.

Siguimos a Tzul (2018) cuando analiza entonces las estrategias sostenidas por las mujeres en la vida comunal en una experiencia concreta, que imprimen la tensión entre conservación y transformación (Tzul 2018: 174) y que, por tanto, son luchas que buscan transformaciones, de un efecto concreto, que impugnan su lugar en las tramas que sostienen la reproducción de la vida. En el caso presentado creemos que la impugnación que están planteando las mujeres indígenas buscan - también hacer un agujero a la realidad que nos domina a todas. Hoy cuando la dominación está desorganizando vía la ruptura de los espacios orgánicos y la jalada de la parcialidad masculina hacia la jerarquización, las luchas de las mujeres están mostrando una disputa por transformar la vida en sus espacios concretos, pero las lianas de sostienen las alianzas son hoy más largas, planteando el movimiento de des-sujetarse de las tramas de dominación des-hilvanandolas e hilándolas de nuevo, desde un nosotras (Gutierrez 2016). 


\section{REFERENCIAS:}

BALZA. 2001. Tierra, Territorio y Territorialidad. Indigena. Un estudio antropologico sobre la evolución en las formas de ocupación del espacio del pueblo indigena chiquitano de ex reduccion jesuita de San José de Chiquitos. Santa Cruz: APCOB/SNV/IWGIA.

BRIADOTTI, R. 2018. Por una política afirmativa. Itinerarios éticos. Barcelona: Gedisa.

DINERSTEIN, A. 2017. Afirmación como Negatividad. Abriendo espacios para otra teoría critica. 25 años del Marxismo Abierto: Reflexiones sobre Teoria Critica y Praxis Revolucionaria. Puebla.

FEDERICI, S. 2004. EI Caliban y la bruja. Mujeres Cuerpos y Acumulación orginaria. . Madrid: Traficantes de sueños.

GIL, S. L. 2011. Nuevos Feminismos. Sentidos comunes en la dispersión. Madrid: Traficantes de sueños.

GUTIERREZ, R. 2014. "Políticas en femenino. Reflexiones acerca de lo femenino moderno y del significado de sus políticas". In: M. Millán, Más Allá del Feminismo. Mexico: Red de feminismos decoloniales. pp. 87-98.

GUTIERREZ, R., LINSALATA, L.; NAVARRO, M. 2016. Repensar lo político, pensar lo común. Claves para la discusión. In: L. L. Daniel Inclán, Modernidades alternativas. Mexico: UNAM. pp. 377-415.

GUTIÉRREZ, R.; SOSA, M. N.; REYES, I. 2018. El entre mujeres como negación de las formas de interdependencia impuestas por el patriarcado capitalista y colonial. Reflexiones en torno a la violencia y la mediación patriarcal. Revista Heterotopías del Área de Estudios del Discurso de FFy: 1-15.

GUTIÉRREZ, R. 2015. A propósito del trabajo de Silvia Federici. Colocar la reproducción material y simbólica de la vida social y la capacidad humana de producir lo común como punto de partida para la reflexión crítica y la práctica política. Apantle. Revista de Estudios Comunitarios 1: 169-176.

GUTIERREZ, R. 2016. Horizonte Comunitario-Popular, antagonismos y produccion de lo comun en America latina. Guatemala: SOCEE.

HERNANDO, A. 2012. La Fantasia de la individualidad. Sobre la construcción sociohistorica del sujeto moderno. Madrid: Katz editores.

LERNER, G. 2017. La Creacion del Patriarcado. Iruñea-Pamplona: Katakrak.

MENENDEZ, M. 2017. Entre mujeres: nuestro deseo de cambiarlo todo. Apantle Revista de Estudios Comunitarios 3: 53-68.

NAVARRO, M. L. 2017. Despojo multiple sobre el tejido de la vida: impactos y resistencias socio- 
ambientales. Revista textual 73.

PATEMAN, C. 2018. EI desorden de las mujeres, Democracia, feminismo y teoria politica. Buenos Aires: Prometeo Libros.

RIVERA, M. M. 2003. Nombrar el mundo en femenino. Barcelona: Icaria editorial.

SEGATO, R. 2016. Patriarcado: Del Borde al Centro. Disciplinamiento, territorialidad y crueldad en la fase apocaliptica del capital. Disponível em: feminisresearchviolence.org

TOMICHA, T.; CUELLAR, C. 2019. Subvertir la dominación: Retos para una práctica y organización feminista popular y diversa en un territorio hostil. In: C. Lopez, L. Gutierrez, \& D. Mokrani. Desplegando nuestro hacer politico. La Paz: Territorio Feminista. pp. 98-116.

TZUL, G. T. 2018. Sistemas de Gobierno Comunal Indígena. Mexico: Libertad Bajo Palabra.

\section{AUTORA:}

\section{Claudia Cuellar}

Boliviana magister en Ciencias Sociales, mención Sociología de la Universidad de Chile. Actualmente cursa el primer año del Doctorado en Sociología de la Benemérita Universidad Autónoma de Puebla, México en el Seminario Entramados Comunitarios y Formas de lo Político.

Email: claudia.cuellarsuarez@gmail.com 